\title{
One approach to solve some problems of management under uncertainty
}

\author{
Teimuraz Tsabadze \\ a) Georgian Technical University \\ b) Georgian American University \\ Tbilisi, Georgia \\ Teimuraz.Tsabadze@yahoo.com
}

\author{
Archil Prangishvili \\ Rector \\ Georgian Technical University \\ Tbilisi, Georgia \\ rectoroffice@gtu.ge
}

\begin{abstract}
We consider the approaches to making decisions for control problems in nonstandard situations with a lack of the previous experience and incomplete knowledge of the considered problem. In such cases we usually cannot do without expert evaluations which lead to the process of group decision-making, and it becomes necessary to solve a problem of alternatives aggregation. It has been proposed to solve such problems by means of fuzzy sets. The approach is based on the coordination index and the similarity of finite collections of fuzzy sets and takes into account the specific character of the fuzzy aggregation operator. The approach is discussed and its algorithm is presented. An example of the application of the proposed method is given.
\end{abstract}

Keywords - Group decision-making; finite collection of fuzzy set; increasing shuffling; coordination index; similarity; fuzzy aggregation operator.

\section{INTRODUCTION}

The modern system of knowledge about management (control) is formed on the basis of various sciences. The management process is a difficult complex system. for example in management of marketing. An important task of management is the integration of all qualities and aspects of the controlled object in order to achieve general purposes set before this object [6] - this means the necessity to analyze a multifactor function whose parameter values are frequently represented by fuzzy or incomplete information which is as a rule is subjective.

The control process is entirely based on the decisionmaking factor.

According to the character of problems, which arise in the course of activity of a controlled object, and methods used for decision-making, we can distinguish the groups of problems $[3,4]$ as shown in the table below:

Structured or, following the terminology of [5], programmed problems - These are solutions which once made become the rules controlling all future actions. They are called structured (programmed) decisions and are part of daily activities of a controlled object, are permanently repeated and comply with the policy adopted by managers. They can be obtained by economical-mathematical methods and the algorithms, already developed, are available in most cases.

Non-structured (non-programmed) problems - A solution should be obtained in a non-standard situation with a lack of previous experience is characterized by incomplete knowledge about the main components and needs non-ordinary approaches. In this area heuristic methods are of great use. Such solutions cause embarrassment in the manager because each time he has to seek for a procedure of their selection. This group includes in particular solutions for a concrete controlled object to get out of crisis, as well as solutions concerning a search for a strategy, determination of an amount of capital to be invested into new production, and so on.

Scientifically substantiated standard problems solved by the statutory rules on solution selection - Scientifically substantiated solutions are those which are adopted on the basis of scientific arguments. They are also called rational because they are supported by scientifically proven schemes incorporating various models, comparisons of different variants and so on. Obtaining such a solution needs much time, the process is frequently iterative, but enables the manager to find a solution.

Intuitive problems and problems based on opinions - As different from scientifically substantiated solutions, intuitive solutions are taken promptly, by feeling that one must act this way and not differently. Such solutions are based on personal experience.

It is obvious that managers encounter big difficulties when they are confronted with non-structured problems (item 2 in the above table), which frequently have to be solved by heuristic methods.

In the present paper, we focus the attention on "the weakest link" - the non-structured process of making management decisions. Our objective is to solve the most frequently occurring problems in situations which lack the previous experience and original information is incomplete. In our opinion confirmed by experience, after the detailed analysis of the management situation the next important step is to find input data to be used in making a final decision. Here, as a rule, we cannot do without experts' evaluations which lead to the process of group decision-making. In this situation, the 
manager (managers) has (have) to solve the problem of alternatives aggregation.

All people usually think in uncertain categories and are not guided only by the "yes" or "no" principle. For example, if the question is asked "Is this project profitable?", classical mathematics answers either "yes" or "no", while the relatively young fuzzy sets theory gives one a chance to model such categories as "unprofitable", "not quite profitable", "profitable", "more profitable", "very profitable" and so on.

In 1965, American scientist L. Zadeh published the paper "Fuzzy Sets" [10], in which he drew the attention of the world scientific community to an absolutely novel direction in the development of mathematics and applied sciences. Instead of the classical membership function of some object in some set ( 0 or 1$)$, Zadeh introduced the continuous membership interval $-[0 ; 1]$.

Fuzzy sets theory made an invaluable contribution to the development of new information-control technologies. It is a powerful instrument of analysis of weakly structured, fragmentary, incomplete and fuzzy information.

The point is that in the absence of a general collection of data, i.e. of a sufficiently large initial database, even such welltested instruments of modeling various situations as probability theory and mathematical statistics are not capable to fairly take into consideration incomplete and fuzzy information [2].

We propose an approach for group-decision making under uncertainty, where experts' opinions are expressed by quantitative values.

\section{ESSENTIAL NOTIONS AND THE THEORETICAL BACKGROUND}

Let $X$ be the universe. A mapping $A: X \rightarrow[0 ; 1]$ is called $a$ fuzzy set on $X$. The value $\mu_{A}(x)$ of $A$ at $x \in X$ denotes the membership degree of $\mathrm{x}$ in $A$. Thus the fuzzy set $A$ can be determined as follows:

$$
A=\left\{\left(x \mid \mu_{A}(x)\right)\right\}, x \in X, \mu_{A}(x) \in[0 ; 1] .
$$

$\Psi(X)=\{\mu \mid \mu: X \rightarrow[0 ; 1]\}$ is the lattice of all fuzzy sets on $X$.

$\varnothing$ is a minimal element of $\Psi(X): \mu_{\varnothing}(x)=0 \quad \forall x \in X$.

$U$ is a maximal element of $\Psi(X): \mu_{U}(x)=1 \quad \forall x \in X$.

$$
\begin{aligned}
& A=B \Leftrightarrow \mu_{A}(x)=\mu_{B}(x) \forall x \in X, \quad A, B \in \Psi(X) . \\
& A \subseteq B \Leftrightarrow \mu_{A}(x) \leq \mu_{B}(x) \forall x \in X, A, B \in \Psi(X) . \\
& A \subset B \Leftrightarrow \mu_{A}(x) \leq \mu_{B}(x) \quad \forall x \in X \text { and } \\
& \exists x_{0} \in X \mid \mu_{A}\left(x_{0}\right)<\mu_{B}\left(x_{0}\right)
\end{aligned}
$$

The union of fuzzy sets $A$ and $B$ is $\mu_{A \cup B}(x)=\max \left\{\mu_{A}(x), \mu_{B}(x)\right\} \forall x \in X$.
The intersection of fuzzy sets $A$ and $B$ is $\mu_{A \cap B}(x)=\min \left\{\mu_{A}(x), \mu_{B}(x)\right\} \quad \forall x \in X$.

It is not difficult to verify that the distributivity of $\bigcap$ and $U$ holds in $\Psi(X)$ :

$$
\begin{aligned}
A \cap(B \cup C) & =(A \cap B) \cup(A \cap C), \\
A \cup(B \cap C) & =(A \cup B) \cap(A \cup C) .
\end{aligned}
$$

We say that the function $v: \Psi(X) \rightarrow \mathfrak{R}^{+}$is the isotone valuation on $\Psi(X)$ if [1]:

$$
v(A \cup B)+v(A \cap B)=v(A)+v(B)
$$

and

$$
A \subseteq B \Rightarrow v(A) \leq v(B)
$$

We say that the isotone valuation $v$ is continuous if for each $a \in[v(\varnothing) ; v(U)]$ there exists

$A \in \Psi(X)$ such that $v(A)=a$.

Let us consider the equation

$$
\rho(A, B)=v(A \cup B)-v(A \cap B) .
$$

We will show that (2.3) represents the metric on $\Psi(X)$, i.e. it meets the following requirements:

1) $\rho(A, B)=0 \Leftrightarrow A=B$;

2) $\rho(A, B)=\rho(B, A)$

3) $\rho(A, C)+\rho(C, B) \geq \rho(A, B), \quad \forall C \in \Psi(X)$.

Proof. Let $\rho(A, B)=0 \Rightarrow v(A \cup B)=v(A \cap B)$. By (2.4) we can easily conclude that $A \cup B=A \cap B \Rightarrow A=B$.

Now let $A=B \Rightarrow A \cup B=A \cap B \Rightarrow v(A \cup B)$

$$
=v(A \cap B) \Rightarrow \rho(A, B)=0
$$

So, 1) is true.

$$
\begin{aligned}
& \rho(A, B)=v(A \cup B)-v(A \cap B) \\
& =v(B \bigcup A)-v(B \cap A)=\rho(B, A)
\end{aligned}
$$

2) is also true.

$$
\begin{aligned}
& \rho(A, B)=v(A \cup B)-v(A \cap B) \\
& \stackrel{(2.2)}{\leq} v((A \cup C) \cup(C \bigcup B))-v((A \cap C) \cap(C \cap B)) \\
& \stackrel{(2.2)}{=} v(A \cup C)+v(C \bigcup B)-v((A \cup C) \cap(C \bigcup B)) \\
& -v(A \cap C)-v(C \cap B)+v((A \cap C) \cup(C \cap B))
\end{aligned}
$$




$$
\begin{aligned}
& \stackrel{(2.1),(2.2)}{=} \rho(A, C)+\rho(C, B)+v(C \cap(A \cup B)) \\
& -v(C \cup(A \cap B)) \stackrel{(2.2)}{\leq} \rho(A, C)+\rho(C, B)
\end{aligned}
$$

Thus 3) has been proved.

$\Psi(X)$ with isotone valuation $v$ and metric (2.3) is called the metric lattice of fuzzy sets.

Definition 2.1[7]. In the metric lattice the fuzzy set $A^{*}$ is the representative of a finite collection of fuzzy sets $\left\{A_{j}\right\}$, $j=\overline{1, m}, \quad m=2,3, \ldots$, if

$$
\sum_{j=1}^{m} \rho\left(A^{*}, A_{j}\right) \leq \sum_{j=1}^{m} \rho\left(B, A_{j}\right), \quad \forall B \in \Psi(X) .
$$

Now let us introduce the concept of increasing shuffling of a finite collection of fuzzy sets.

Definition 2.2[7]. A finite collection of fuzzy sets $\left\{A_{j}^{\prime}\right\}$ is the increasing shuffling of a finite collection of fuzzy sets $\left\{A_{j}\right\}$ if for each $x \in X$ the finite sets $\left\{\mu_{A_{j}}(x)\right\}$ and $\left\{\mu_{A_{j}}(x)\right\}$ are equal to each other and $\mu_{A_{1}^{\prime}}(x) \leq \mu_{A_{2}^{\prime}}(x) \leq \ldots \leq \mu_{A_{m}^{\prime}}(x)$, $j=\overline{1, m}, \quad m=2,3, \ldots$.

Thus the increasing shuffling represents a finite collection of nested fuzzy sets. The equality

$$
\sum_{j=1}^{m} \rho\left(B, A_{j}\right)=\sum_{j=1}^{m} \rho\left(B, A_{j}^{\prime}\right)
$$

holds in the metric lattice for any $B \in \Psi(X)$ and a finite collection of fuzzy sets $\left\{A_{j}\right\}, j=\overline{1, m}, \quad m=2,3, \ldots$.

Theorem 2.1[7]. In the metric lattice of fuzzy sets the representative $A^{*}$ of a finite collection of fuzzy sets $\left\{A_{j}\right\}, j=\overline{1, m}, \quad m=2,3, \ldots$, is determined as follows:

$$
\begin{gathered}
A_{m / 2}^{\prime} \subseteq A^{*} \subseteq A_{m / 2+1}^{\prime} \quad \text { if } m \text { is even; } \\
A^{*}=A_{(m+1) / 2}^{\prime} \quad \text { if } m \text { is odd }
\end{gathered}
$$

Here and further the symbol [ ] denotes the integer part of a number.

We say that a finite collection of fuzzy sets is symmetrical [7] if in its increasing shuffling the first [ $(2 m+1) / 4]$ sets are equal to $\varnothing$ and the last $[(2 m+1) / 4]$ sets are equal to $U$, $m=2,3, \ldots$.

Definition 2.3[7]. In a metric lattice of fuzzy sets the functional

$$
S: \underbrace{\Psi(X) \times \ldots \times \Psi(X)}_{m \text { times }} \rightarrow \mathbb{R}^{+}
$$

is the coordination index of the finite collection of fuzzy sets $\left\{A_{j}\right\}, j=\overline{1, m}, m=2,3, \ldots, \quad$ if it satisfies the following postulates:

P1. $S\left\{A_{j}\right\}=0$ if and only if the finite collection of fuzzy sets $\left\{A_{j}\right\}$ is symmetrical;

P2. $S\left\{A_{j}\right\}$ reaches a maximal value if and only if all fuzzy sets of the finite collection are equal to one another;

P3. $S\left\{A_{j}\right\} \geq S\left\{B_{j}\right\} \quad$ if $\quad \sum_{j=1}^{m} \rho\left(A^{*}, A_{j}\right) \leq \sum_{j=1}^{m} \rho\left(B^{*}, B_{j}\right)$; moreover, $S\left\{A_{j}\right\}=S\left\{B_{j}\right\}$ if and only if $\sum_{j=1}^{m} \rho\left(A^{*}, A_{j}\right)=\sum_{j=1}^{m} \rho\left(B^{*}, B_{j}\right)$;

P4. $S\left\{A_{j} \cup B_{j}\right\}+S\left\{A_{j} \cap B_{j}\right\}=S\left\{A_{j}\right\}+S\left\{B_{j}\right\}$.

Setting aside the question of independence and completeness of the postulates introduced above (Definition 2.3), by constructing the corresponding example [7] we show that this axiomatic is consistent.

Theorem 2.2[7]. In the metric lattice of fuzzy sets the functional $S\left\{A_{j}\right\}$ is the coordination index of the finite collection of fuzzy sets $\left\{A_{j}\right\}, j=\overline{1, m}, m=2,3, \ldots$, if

$S\left\{A_{j}\right\}=q\left(\rho(\varnothing, U)-[(2 m+1) / 4]^{-1} \times \sum_{j=1}^{m} \rho\left(A^{*}, A_{j}\right)\right), q>0$

Moreover, if the isotone valuation $v$ is continuous, this representation is unique.

It is obvious that

$$
S_{\max }=q \rho(\varnothing, U), \quad \theta>0 .
$$

A method of fuzzy aggregation based on group expert evaluations is presented in [8]. Below we give the basic theoretical results and explanations needed to understand the principles and work of this method. First, we introduce the important concept of similarity for finite collections of fuzzy sets, where the metric approach is used.

Definition 2.4[8]. In the metric lattice of fuzzy sets the finite collection of fuzzy sets $\left\{A_{j}\right\}$ is similar to the finite collection of fuzzy sets $\left\{B_{j}\right\}$ if for each $x \in X$ $\rho\left(A_{i}^{\prime}, A_{i-1}^{\prime}\right)=k \rho\left(B_{i}^{\prime}, B_{i-1}^{\prime}\right), i=\overline{2, m}, j=\overline{1, m}, m=2,3, \ldots$,

where $k>0$ is the similarity coefficient, $\left\{A_{j}^{\prime}\right\},\left\{B_{j}^{\prime}\right\}$ are the increasing shufflings of $\left\{A_{j}\right\}$ and $\left\{B_{j}\right\}$ respectively.

We denote the similarity of two finite collections of fuzzy sets in the metric lattice of fuzzy sets by $\left.\left\{A_{j}\right\} \stackrel{k}{\cong}\left\{B_{j}\right\} \Leftrightarrow\left\{B_{j}\right\} \cong A_{j}\right\}$ or simply by $\left\{A_{j}\right\} \cong\left\{B_{j}\right\}$.

One way of constructing such a finite collection of fuzzy sets in the metric lattice with continuous isotone valuation $v$ is presented in [8]. Let $\left\{B_{j}\right\}, j=\overline{1, m}, m=2,3, \ldots$, be a finite 
collection of fuzzy sets. To construct a finite collection of fuzzy sets $\left\{C_{j}\right\}$ similar to $\left\{B_{j}\right\}$, the following general formula are used:

$$
\begin{aligned}
& \forall x \in X \quad \mu_{C_{j}}(x)=k \rho\left(B_{1}^{\prime}, B_{j}^{\prime}\right)+\mu_{C_{1}}(x), \\
& j=\overline{1, m}, m=2,3, \ldots, \mu_{C_{1}}(x)=v\left(C_{1}\right)=\alpha \mid 0 \leq \alpha<1
\end{aligned}
$$

Now we present an important theorem. We remind that the maximal coordination index $S_{\max }$ of a finite collection of fuzzy sets is determined by (2.7).

Theorem 2.3 [8]. If $\left\{A_{j}\right\} \stackrel{k}{\cong}\left\{B_{j}\right\}$, then for the coordination indices of these two finite collections of fuzzy sets the equality $S\left\{A_{j}\right\}=k S\left\{B_{j}\right\}+(1-k) S_{\max }, j=\overline{1, m} m=2,3, \ldots$ holds.

Corollary. $\left\{A_{j}\right\} \stackrel{1}{\cong}\left\{B_{j}\right\} \Rightarrow S\left\{A_{j}\right\}=S\left\{B_{j}\right\}, j=\overline{1, m}, m=2,3 \ldots$

The following theorem is the main theoretical basis of our fuzzy aggregation method.

Theorem 2.4 [8]. In the metric lattice of fuzzy sets with continuous isotone valuation for any two finite collection of fuzzy sets $\left\{A_{j}\right\}$ and $\left\{B_{j}\right\}$ such that $S\left\{A_{j}\right\}, S\left\{B_{j}\right\}<S_{\max }$ there exists a finite collection of fuzzy sets $\left\{C_{j}\right\}$ such that $\left\{C_{j}\right\} \cong\left\{B_{j}\right\}$ and $S\left\{C_{j}\right\}=S\left\{A_{j}\right\}, j=\overline{1, m}, \quad m=2,3, \ldots$.

The next theorem determines some specific conditions for uniqueness of finite collection of fuzzy sets, which is similar to the given one.

Theorem 2.5 [8]. In the metric lattice of fuzzy sets with continuous isotone valuation for any two finite collections of fuzzy sets such as $\left\{C_{j}\right\} \stackrel{k}{\cong}\left\{B_{j}\right\}$ and $S\left\{C_{j}\right\}>S\left\{B_{j}\right\}$, for each $k$ there exists a unique finite collection of fuzzy sets $\left\{A_{j}\right\}$ such that $\quad\left\{A_{j}\right\} \cong\left\{C_{j}\right\} \quad$ and $\quad A_{l}=B_{l}^{\prime}, j=\overline{1, m}$, $l \in\{1,2, \ldots, m\}, \quad m=2,3 \ldots$.

By Theorem 2.5 we obtain the general formula

$$
\begin{aligned}
& \mu_{A_{l j}}(x)=\mu_{B_{l}^{\prime}}(x)+k\left(v\left(B_{j}^{\prime}\right)-v\left(B_{l}^{\prime}\right)\right), \\
& j=\overline{1, m}, \quad l \in\{1,2, \ldots, m\}, \quad \forall x \in X
\end{aligned}
$$

In the sequel, we will need one specific modification of (2.9). Under the conditions of Theorem 2.5 let us consider the finite collection of fuzzy sets $\left\{\bar{A}_{j}\right\}$ whose membership functions are the arithmetic means of the respective membership functions (2.9):

$$
\left\{\bar{A}_{j}\right\}=\left\{\frac{\sum_{l=1}^{m} \mu_{A_{j}}(x)}{m}\right\}, j=\overline{1, m}, m=2,3, \ldots, \forall x \in X .
$$

$$
\begin{gathered}
\text { From the last expression, by } \\
\left\{\bar{A}_{j}\right\}=\left\{\frac{\sum_{l=1}^{m}\left(\mu_{B_{l}^{\prime}}(x)-k v\left(B_{l}^{\prime}\right)\right)}{m}+k v\left(B_{j}^{\prime}\right)\right\} \text { and using }
\end{gathered}
$$

the notation

$$
c=\frac{\sum_{l=1}^{m}\left(\mu_{B_{l}^{\prime}}(x)-k v\left(B_{l}^{\prime}\right)\right)}{m},
$$

we obtain that

$$
\left\{\bar{A}_{j}\right\}=\left\{c+k v\left(B_{j}^{\prime}\right)\right\}, j=\overline{1, m}, l \in\{1,2, \ldots, m\}, m=2,3, \ldots, \forall x \in X
$$

It is obvious that for $\forall x \in X \quad\left\{\bar{A}_{j}\right\}$ is a finite collection of nested fuzzy sets. From (2.1), (2.10), (2.12), Definition 2.4 and Theorem 2.5 it follows that $\rho\left(\bar{A}_{i}, \bar{A}_{i-1}\right)=v\left(\bar{A}_{i}\right)-v\left(\bar{A}_{i-1}\right)=c+k v\left(B_{i}^{\prime}\right)-c-k v\left(B_{i-1}^{\prime}\right)$ $=k \rho\left(B_{i}^{\prime}, B_{i-1}^{\prime}\right)=\rho\left(C_{i}, C_{i-1}\right) \Rightarrow\left\{\bar{A}_{j}\right\} \cong \stackrel{1}{\cong}\left\{C_{j}\right\}$ $i=\overline{2, m}, j=\overline{1, m}, \forall x \in X$. According to the corollary of Theorem 2.3, $S\left\{\bar{A}_{j}\right\}=S\left\{C_{j}\right\}$.

Thus we have proved

Proposition 2.1[8]. If under the conditions of Theorem 2.5 the finite collection of fuzzy sets $\left\{\bar{A}_{j}\right\}$ is determined by (2.10), then $\left\{\bar{A}_{j}\right\} \cong \stackrel{1}{\cong}\left\{C_{j}\right\}$ and, consequently, $S\left\{\bar{A}_{j}\right\}=S\left\{C_{j}\right\}, j=\overline{1, m}$, $m=2,3, \ldots, \forall x \in X$.

For the realization of the proposed method we need a specific aggregation operator that meets certain requirements. Presently, in the fuzzy set theory there are several well known fuzzy aggregation operators (see e.g. [9]). The representative of a finite collection of fuzzy sets determined by Definition 2.1 is a new kind of fuzzy aggregation operator. By Theorem 2.1 the representative of a finite collection of fuzzy sets $\left\{A_{j}\right\}$, $j=\overline{1, m}, \quad m=2,3, \ldots, \quad$ yields the expression $A_{m / 2}^{\prime} \subseteq A^{*} \subseteq A_{m / 2+1}^{\prime}$ when $m$ is even or $A^{*}=A_{(m+1) / 2}^{\prime}$ when $m$ is odd. This means that if $m$ is even and $A_{m / 2}^{\prime} \subset A_{m / 2+1}^{\prime}$, then the representative can take an infinite number of values. In [8], after the detailed discussion and justification, the following form of a unique representative is defined (here and in the sequel we use the notation $\mu_{A}$ instead of $\left.\mu_{A}(x), \forall x \in X\right)$ : 


$$
\mu_{A}=\left\{\begin{array}{l}
\left(\mu_{\dot{A}_{m / 2]}}+\mu_{\dot{A}_{(m+3) / 2]}}\right) / 2 \text { if } \sum_{j=1}^{[(m+1) / 2]} \rho\left(\dot{A}_{j}, \dot{A}_{m / 2]}\right)=\sum_{j=[m / 2]+1}^{m} \rho\left(\dot{A}_{j}, \dot{A}_{(m+3) / 2]}\right), \\
\sum_{\dot{A}_{m / 2]}}+\frac{\sum_{j=1}^{[(m+1) / 2]} \rho\left(\dot{A}_{j}, \dot{A}_{m / 2]}\right)}{\sum_{j=1}^{[(m+1) / 2]} \rho\left(\dot{A}_{j}, \dot{A}_{m / 2]}\right)+\sum_{j=[m / 2]+1}^{m} \rho\left(\dot{A}_{j}, \dot{A}_{(m+3) / 2]}\right)}\left(\mu_{\dot{A}_{(m+3) / 2]}}-\mu_{\dot{A}_{m / 2]}}\right) \text { otherwise. }
\end{array}\right.
$$

We finish our theoretical background by determining one specific modification of the introduced fuzzy aggregation operator, which is obtained from the interrelation between the sums in (2.13) and the condition of similarity of two finite collections of fuzzy sets.

Lemma 2.1 [8] If $\left\{A_{j}\right\} \stackrel{k}{\cong}\left\{B_{j}\right\}, \quad j=\overline{1, m}, \quad m=2,3, \ldots$ then $\sum_{j=1}^{[(m+1) / 2]} \rho\left(A_{j}^{\prime}, A_{[m / 2]}^{\prime}\right)=k \sum_{j=1}^{[(m+1) / 2]} \rho\left(B_{j}^{\prime}, B_{[m / 2]}^{\prime}\right)$
$\sum_{j=[m / 2]+1}^{m} \rho\left(A_{j}^{\prime}, A_{[(m+3) / 2]}^{\prime}\right)=k \sum_{j=[m / 2]+1}^{m} \rho\left(B_{j}^{\prime}, B_{[(m+3) / 2]}^{\prime}\right)$.

Assume that the finite collection of fuzzy sets $\left\{\bar{A}_{j}\right\}$ is given by (2.10). We want to determine $\mu_{\bar{A}_{[m / 2]}}$ and $\mu_{\bar{A}_{[(m+3) / 2\}}}$. By (2.12) and (2.11) we easily obtain that

$$
\mu_{\bar{A}_{[m / 2]}}=c+k v\left(B_{[m / 2]}^{\prime}\right) ; \mu_{\bar{A}_{[(m+3) / 2]}}=c+k v\left(B_{[(m+3) / 2]}^{\prime}\right)
$$

Using (2.3) and (2.14) it is easy to check that the equality

$$
\mu_{\bar{A}_{[(m+3) / 2]}}-\mu_{\bar{A}_{[m / 2]}}=k \rho\left(B_{[m / 2]}^{\prime}, B_{[(m+3) / 2]}^{\prime}\right)
$$

holds.

Finally, if the conditions of Theorem 2.5 are satisfied, $\left\{\bar{A}_{j}\right\}$ is given by (2.10) and $\left\{\bar{A}_{j}\right\} \stackrel{k}{\cong}\left\{B_{j}^{\prime}\right\}$, then by Lemma $2.1,(2.14)$ and (2.15) we can rewrite formula (2.13) as

$$
\mu_{A_{j}}=\left\{\begin{array}{l}
c+k \frac{v\left(B_{m / 2]}^{\prime}\right)+v\left(B_{[(m+3) / 2]}^{\prime}\right)}{2} \text { if } \sum_{j=1}^{[(m+1) / 2]} \rho\left(B_{j}^{\prime}, B_{[m / 2]}^{\prime}\right)=\sum_{j=[m / 2]+1}^{m} \rho\left(B_{j}^{\prime}, B_{(m+3) / 2]}^{\prime}\right), \\
c+k\left(v\left(B_{[m / 2]}^{\prime}\right)+\frac{\rho\left(B_{m / 2]}^{\prime}, B_{(m+3) / 2]}^{\prime}\right) \sum_{j=1}^{[(m+1) / 2]} \rho\left(B_{j}^{\prime}, B_{[m / 2]}^{\prime}\right)}{\sum_{j=1}^{m} \rho\left(B_{j}^{\prime}, B_{m / 2]}^{\prime}\right)+\sum_{j=[m / 2]+1}^{m} \rho\left(B_{j}^{\prime}, B_{(m+3) / 2]}^{\prime}\right),}\right. \text { otherwise. }
\end{array}\right.
$$

\section{DESCRIPTION OF THE FUZZY AGGREGATION METHOD AND ITS ALGORITHM}

It is assumed here that all experts have the same qualification (otherwise see Remark 3.1). Let the group of experts evaluate the membership degree of the fuzzy object in the given universe. As a result of this evaluation we obtain a finite collection of fuzzy sets and proceed to the process of group decision-making. We try to construct the fuzzy set of the obtained finite collection of fuzzy sets. Assume that we can calculate the coordination index of the obtained finite collection of fuzzy sets at each element of the universe. It is clear that the experts' evaluations at each element form a finite collection of one-element fuzzy sets. If there existed several elements, where the coordination index reaches its maximal value, then by (2.6) all members of the finite collection of oneelement fuzzy sets would be equal to one another. This means that then the experts' work is ideal and each of the fuzzy sets constructed by them is the only result of group decisionmaking. But by the nature of fuzziness such a theoretical chance may occur very rarely.

We are interested in the cases where the coordination index does not reach its maximal value. Suppose that the greatest value of the coordination index is reached at one element of the universe (there may also be a few elements). In that case we consider it to be the experts' best attempt! They are not automata and cannot act with invariably constant concentration on each element of the universe. Let us take an analogy from sport. In such kinds of sports as long and high jumps, discus throwing, weight lifting and so on a sportsman is evaluated by his best result in a finite series of attempts. In our case we consider that this element is the point of maximal coordination of the experts and suppose that they can potentially act with the same coordination at all other elements of the universe. We obtain the result of fuzzy aggregation at the point of maximal coordination by using operator (2.13). Let us try to map this best attempt onto the other elements of the universe.

The way of implementing this idea is as follows. We choose an element of the universe where the value of the coordination index is smaller than the greatest value. If the experts' evaluations at this element are not a finite collection of nested one-element fuzzy sets, then we operate with its increasing shuffling. Thus, without loss of generality this finite collection can be assumed to consist of nested one-element fuzzy sets. Let us construct such a finite collection of oneelement fuzzy sets that is "similar" to the above-mentioned finite collection of nested one-element sets and at the same time has the greatest coordination index. Now let us discuss the notion of "similarity" in informal terms.

We can imagine a finite collection of one-element fuzzy sets as a finite set of geometric points with equal abscissae, while their ordinates as representing the evaluations of experts at this element of the universe. Then any finite collection of one-element fuzzy sets similar to the above-mentioned one can be represented by the same number of points with equal abscissae, while the distances between the neighboring ordinates are proportional to the respective distances of the finite collection of one-element fuzzy sets. In the other words, in a similar finite collection of one-element fuzzy sets the ordinates are close to or distant from one another, preserving at the same time the proportionality of the respective distances. The strict mathematical determination of the similarity for two finite collections of fuzzy sets is given in Definition 2.4. Formula (2.8) allows us to construct a finite collection of one- 
element fuzzy sets similar to the finite collection of nested oneelement sets.

From Definition 2.4 and formula (2.8) it follows that there exists an infinite set of finite collections of one-element fuzzy sets similar to the given one. As mentioned above, we have to choose a finite collection of one-element fuzzy sets, the coordination index of which is equal to the coordination index of the finite collection of one-element fuzzy sets constructed at the point of maximal coordination. By Theorem 2.4 we can construct a finite collection of one-element fuzzy sets which is similar to the given one and, at the same time, has the greatest coordination index. But even in that case there exists an infinite set of such finite collections of one-element fuzzy sets. Here the problem reduces to determining the unique finite collection of one-element fuzzy sets which takes into proper account the opinions of all experts pari passu.

Not to "offend" anyone of the experts we act the following way: choose from this infinite set such a finite collection of one-element fuzzy sets where the first one-element fuzzy set is equal to the first one-element fuzzy set of the given finite collection of one-element fuzzy sets; next choose such a finite collection of one-element fuzzy sets where the second oneelement fuzzy set is equal to the second one-element fuzzy set of the given finite collection of one-element fuzzy sets and so on; finally, choose such a finite collection of one-element fuzzy sets where the last one-element fuzzy set is equal to the last one-element fuzzy set of the given finite collection of oneelement fuzzy sets. It is obvious that the number of constructed finite collections of one-element fuzzy sets is equal to the number of experts.

In the sequel we will apply the mapping $\mu: X \rightarrow[0 ; b] \subset \mathbb{R}$ (here we use the membership function with interval $[0 ; b]$ instead of the standard interval standard $[0 ; 1]$ in order to make the examples more obvious).

Remark 3.1. The method can also be applied in the case of different qualification of experts (for example by using weight coefficients). The essence and structure of the proposed approach will not be affected.

Let us summarize briefly the stages of the proposed method and give its formal algorithm.

Let $\Psi(X)=\{\mu \mid \mu: X \rightarrow[0 ; b] \subset \mathfrak{R}\}$ be the metric lattice with continuous isotone valuation $v$, the universe $X$ be the finite set $\left\{x_{1}, x_{2}, \ldots, x_{N}\right\}$ and the group of $m$ experts evaluate the degree of membership of the concept $B$ in the universe. As a result we have the finite collection of fuzzy sets: $\left\{B_{j}\right\}$, $j=\overline{1, m}, \quad m=2,3, \ldots$.

First we construct its increasing shuffling $\left\{B_{j}^{\prime}\right\}$ and under assumption $q=1$ compute $S_{\max }$ by (2.5). After that we compute the coordination index value at each element of the universe by (2.4). Here we have several alternatives.

Assume that at some element the value of this index is maximal (there can be several such elements). This means that each of $m$ constructed fuzzy sets is the result of fuzzy aggregation for these elements.

Now consider only such values of coordination indices that are smaller than the greatest value. Choose among them such an element, where the coordination index value is the greatest (there can be several such elements). It is the point of maximal coordination. Denote by $\left\{A_{j}\right\}$ the finite collection of oneelement fuzzy sets at this element. In this case the result of fuzzy aggregation is obtained by (2.13).

Now consider such elements where the coordination index values are smaller than the greatest value. Let demonstrate the operation of the algorithm at one of these elements. Suppose that at an element $x \in X$ we have the finite collection of oneelement fuzzy sets $\left\{B_{j}^{\prime}\right\}$. Then by Theorem 2.4 we construct at this element a finite collection of one-element fuzzy sets $\left\{C_{j}\right\}$ such that $\left\{C_{j}\right\} \cong\left\{B_{j}^{\prime}\right\}$ and $S\left\{C_{j}\right\}=S\left\{A_{j}\right\}, j=\overline{1, m}, \quad m=2,3, \ldots$. We know that there exists an infinite set of such finite collections of one-element fuzzy sets. Theorem 2.5 allows us to choose from this set exactly such $m$ finite collections of oneelement fuzzy sets where each of them has common elements with $\left\{B_{j}^{\prime}\right\}$. Taking into account the opinion of each expert, we take as a result such a finite collection of one-element fuzzy sets where each member is the arithmetic mean of respective members of these $m$ unique finite collections of one-element fuzzy sets (we remind that each member is a real number). To obtain this finite collection of one-element fuzzy sets we use formula (2.10). By Proposition 2.1 this finite collection of oneelement fuzzy sets is similar to $\left\{C_{j}\right\}$ with the similarity coefficient $k=1$ and, by Corollary of Theorem 2.3, has the greatest coordination index.

Thus the constructed finite collection of one-element fuzzy sets satisfies the conditions of Proposition 2.1 and we obtain the result of fuzzy aggregation by operator (2.16). In the same way one can obtain a fuzzy aggregation operator at any other element of the given universe.

Remark 3.2. We do not need to carry out long and tedious calculations for constructing $m$ new finite collections of oneelement fuzzy sets with each of them having common elements with $\left\{B_{j}^{\prime}\right\}$ and determining their arithmetic means. As a matter of fact, via the coefficient of similarity we operate only with the given finite collection of one-element fuzzy sets $\left\{B_{j}^{\prime}\right\}$. In our opinion it is an effective and nice circumstance.

Now we present the generalized algorithm of the fuzzy aggregation method.

\section{Algorithm}

Step 0: Initialization: the finite collection of one-element fuzzy sets $\left\{B_{j}\right\}, \quad$ its increasing shuffling $\left\{B_{j}^{\prime}\right\}, j=\overline{1, m}, m=2,3, \ldots$. Denote the result of the fuzzy aggregation in element $x_{i}, i=\overline{1, N}$ by $\mu\left(x_{i}\right)$.

Step 1: Compute the values of coordination indices of the finite collection of one-element fuzzy sets $\left\{B_{j}^{\prime}\right\}$ at each element 
$x_{i}, i=\overline{1, N}$ by (2.4). Denote these values by $S\left(x_{1}\right), S\left(x_{2}\right), \ldots, S\left(x_{N}\right)$ respectively. Compute the value of $S_{\max }$ by (2.5).

Step 2: Choose from the set $\left\{S\left(x_{i}\right)\right\}$ such an element $S^{*}$ which is greater than or equal to any other element except $S_{\max }$.

Step 3: Do Step 4 for $i=\overline{1, N}$.

Step 4: Compute $\Delta=S^{*}-S\left(x_{i}\right)$ :

If $\Delta<0$ then $\mu\left(x_{i}\right)=\mu_{B_{j}}\left(x_{i}\right)$;

If $\Delta=0$ then compute the value of $\mu\left(x_{i}\right)$ by (2.13);

If $\Delta>0$ then compute the value of $k_{i}$ from the equation $S^{*}=k_{i} S\left(x_{i}\right)+\left(1-k_{i}\right) S_{\max }$ and the value of $\mu\left(x_{i}\right)$ by (2.11) and (2.16).

Step 5: Representation is $\left\{\mu\left(x_{1}\right), \mu\left(x_{2}\right), \ldots, \mu\left(x_{N}\right)\right\}$.

\section{ILLUSTRATIONS}

Here we give an example of the practical application of the introduced method. Suppose that we have some new project (from an arbitrary area). In the first place the parameterization of the project is carried out, i.e. the components (and their measured values) of the project vector are determined. Some of these parameters can be already determined and their values will be used as they are in the final project decision. To determine the final values of parameters with incomplete initial data it is necessary to invite a group of experts. The task of the project manager (managers) is to obtain the experts' evaluations of each uncertain (fuzzy) parameter and on their basis to adopt the final decision.

The manager offers the group of experts to give a numerical evaluation of each component of the project vector. Suppose that the project suggests the construction of a new hydroelectric power station. Then the following parameters may serve as examples of uncertain data of the project vector:

$x_{k}$ is the operating costs. They directly depend on the predicted quantity of distributed electric power, on the varying prices of fuel, lubricant, transport, various kinds of maintenance and so on. Hence it can be stated that this parameter possesses a high degree of uncertainty;

$x_{l}$ is the rate of profit (return) on the equity capital. In all cases this parameter possesses some degree of uncertainty because profit is a predicted value;

$x_{m}$ is the loan capital share in the total capital amount. In all cases this parameter depends on many variable values such as an interest rate of credit auctions, an interest rate of a bank loan, an interest rate of a bank deposit, a risk factor, specifications of an investment program (e.g. the invested capital cost) and so on. Therefore this parameter contains elements of uncertainty;

$x_{n}$ is the quantity of distributed electric power. This value is taken from the capital balance, but the balance itself is predictable and besides this value is influenced by the chanding demand as well as by other market factors. Hence this parameter contains elements of uncertainty ; and so on.

After obtaining the subjective evaluations of the experts, i.e. the numerical values of the above-listed parameters of the vector, these values are projected onto the real interval interval $[0 ; \mathrm{b}]$ by means of the normalizing coefficients . Assume that the vector consists of $\mathrm{N}$ components and we have $\mathrm{m}$ experts. In that case a finite collection of fuzzy sets is formed, which consists of $\mathrm{m}$ sets, each of them containing $\mathrm{N}$ elements $\left\{A_{j}\right\}, j=\overline{1, m}, m=2,3, \ldots$.

Now we must obtain the resulting vector of the components of the considered project. Let us describe how we can do this.

Let the universe $X$ be a finite set $\left\{x_{1}, x_{2}, \ldots, x_{N}\right\}, N \geq 1$, the metric lattice $\Psi(X)=\{\mu \mid \mu: X \rightarrow[0 ; b] \subset \mathbb{R}\}$ and the isotone valuation $v(A)=\sum_{i=1}^{N} \mu_{A}\left(x_{i}\right), A \in \Psi(X)$. It is obvious that $v(A \cup B)+v(A \cap B)=v(A)+v(B) \quad$ and $A \subseteq B \Rightarrow v(A) \leq v(B)$.

It is easy to see that this isotone valuation $v$ is continuous (see Section 2).

By (2.3) the valuation $v$ determines the following metric:

$$
\rho(A, B)=\sum_{i=1}^{N}\left|\mu_{A}\left(x_{i}\right)-\mu_{B}\left(x_{i}\right)\right|, x_{i} \in X, A, B \in \Psi(X)
$$

Note that in this case the isotone valuation is the $\sum$ Count, while the metric coincides with the Hamming distance.

Using metric (4.1), the formula for determining the coordination index of a finite collection of fuzzy sets (2.6) takes the form

$$
S\left\{A_{j}\right\}=q\left(b N-[(2 m+1) / 4]^{-1} \sum_{j=1}^{m} \sum_{i=1}^{N} \mid \mu_{A^{*}}\left(x_{i}\right)-\mu_{A_{j}}\left(x_{i}\right)\right) \cdot q>0
$$

Without the loss of generality it can be assumed that the coordination index of a finite collection of fuzzy sets changes in the interval $[0 ; 1]$. Then by (4.2) the coordination index of the finite collection of fuzzy sets $\left\{A_{j}\right\}$ yields the expression

$$
S\left\{A_{j}\right\}=\frac{1}{b N}\left(b N-[(2 m+1) / 4]^{-1} \sum_{j=1}^{m} \sum_{i=1}^{N}\left|\mu_{A^{*}}\left(x_{i}\right)-\mu_{A_{j}}\left(x_{i}\right)\right|\right)
$$

It is clear that $S_{\max }=1$.

Now let us evaluate the coordination index of the finite collection of fuzzy sets at each element of the universe. From (4.3) it follows that the coordination index yields the expression

$$
S\left\{A_{j}\right\}=\frac{1}{b}\left(b-[(2 m+1) / 4]^{-1} \sum_{j=1}^{m}\left|\mu_{A^{*}}\left(x_{i}\right)-\mu_{A_{j}}\left(x_{i}\right)\right|\right), \forall x_{i} \in X
$$


By Theorem 2.3 we determine the respective similarity coefficient $k_{i}$ at each element of the universe:

$$
k_{i}=\left(1-S^{*}\right) /\left(1-S\left(x_{i}\right)\right)
$$

It is easy to see that for our case (2.11) takes the form

$$
c_{i}=\frac{1-k_{i}}{m} \sum_{j=1}^{m} \mu_{B_{j}^{\prime}}\left(x_{i}\right)
$$

and in (2.16)

$$
v\left(B_{j}^{\prime}\right)=\mu_{B_{j}^{\prime}}\left(x_{i}\right), \quad \forall x_{i} \in X .
$$

We give the following concrete numerical example. Let the universe $X$ be a finite set $\left\{x_{1}, x_{2}, \ldots, x_{7}\right\}$ and $\Psi(X)=\{\mu \mid \mu: X \rightarrow[0 ; 10]\}$ be a metric lattice with continuous isotone valuation $v(B)=\sum_{i=1}^{7} \mu_{B}\left(x_{i}\right)$. Assume that we have a group of five experts, each of whom evaluated numerically seven uncertain parameters (components) of the project vector $B$. Then we project these values onto the real interval $[0 ; 10]$. As a result we obtain the finite collection of fuzzy sets $\left\{B_{j}\right\}, j=\overline{1,5}$ :

$$
\begin{array}{cccccccc} 
& x_{1} & x_{2} & x_{3} & x_{4} & x_{5} & x_{6} & x_{7} \\
B_{1} & 1.0 & 8.0 & 6.4 & 4.8 & 2.0 & 7.2 & 3.0 \\
B_{2} & 1.5 & 7.4 & 6.5 & 3.2 & 4.0 & 8.1 & 3.4 \\
B_{3} & 2.1 & 8.2 & 7.0 & 5.0 & 3.0 & 6.8 & 3.0 \\
B_{4} & 2.5 & 9.0 & 6.7 & 5.5 & 2.6 & 8.2 & 5.0 \\
B_{5} & 1.9 & 9.5 & 6.8 & 3.5 & 2.5 & 8.5 & 5.5
\end{array}
$$

and by Definition 2.2 determine its increasing shuffling $\left\{B_{j}^{\prime}\right\}$ :

$$
\begin{array}{cccccccc} 
& x_{1} & x_{2} & x_{3} & x_{4} & x_{5} & x_{6} & x_{7} \\
B_{1}^{\prime} & 1.0 & 7.4 & 6.4 & 3.2 & 2.0 & 6.8 & 3.0 \\
B_{2}^{\prime} & 1.5 & 8.0 & 6.5 & 3.5 & 2.5 & 7.2 & 3.0 \\
B_{3}^{\prime} & 1.9 & 8.2 & 6.7 & 4.8 & 2.6 & 8.1 & 3.4 \\
B_{4}^{\prime} & 2.1 & 9.0 & 6.8 & 5.0 & 3.0 & 8.2 & 5.0 \\
B_{5}^{\prime} & 2.5 & 9.5 & 7.0 & 5.5 & 4.0 & 8.5 & 5.5
\end{array}
$$

Simplify (4.4) for our case:

$$
S\left\{B_{j}^{\prime}\right\}=0.1 \times\left(10-0.5 \times \sum_{j=1}^{5}\left|\mu_{B^{*}}\left(x_{i}\right)-\mu_{B_{j}^{\prime}}\left(x_{i}\right)\right|\right), \quad \forall x_{i} \in X .
$$

Note that here the representative $B^{*}$ is easily determined by Theorem 2.1. Using the last expression we calculate the values of the coordination index of the increasing shuffling at each element of the universe:

$$
\begin{array}{ccccccc}
x_{1} & x_{2} & x_{3} & x_{4} & x_{5} & x_{6} & x_{7} \\
0.895 & 0.845 & 0.955 & 0.810 & 0.875 & 0.865 & 0.775
\end{array}
$$

The coordination index does not reach its maximal value at every element of the universe. Denote the greatest value by $S^{*}$ $=0.955$.

By (4.5) and (4.10) we obtain $k_{1} \approx 0.4286$, and. by (4.6) and (4.9) $c_{1} \approx 1.0286$. Now by (2.16) and (4.9) with (4.7) taken into account we compute $\mu\left(x_{1}\right) \approx 1.8257$. The fuzzy aggregation at all other elements of the universe is obtained analogously. As a result we have

$\begin{array}{cccccccc} & x_{1} & x_{2} & x_{3} & x_{4} & x_{5} & x_{6} & x_{7} \\ k_{i} \approx & 0.4286 & 0.2903 & 1.0 & 0.2368 & 0.36 & 0 .(3) & 0.2 \\ c_{i} \approx & 1.0286 & 5.9755 & - & 3.3579 & 1.8048 & 5.1733 & 3.184 \\ \mu\left(x_{i}\right) \approx & 1.8257 & 8.4087 & 6.65 & 4.4340 & 2.7588 & 7.6518 & 3.848\end{array}$

We know that all experts have the same qualification. We may find out which of them had the highest concentration of evaluation effort in the course of evaluations. For this we have to calculate a standard deviation of the evaluations from the result of the group decision-making for each expert:

$$
\delta_{j}=\left(\sum_{i=1}^{7}\left|\mu\left(x_{i}\right)-\mu_{B_{j}}\left(x_{i}\right)\right|\right) / 7 \text {. }
$$
obtain

Using (4.8), (4.9) and (4.11), from the last expression we

$$
\begin{array}{cccccc}
\text { No. } & 1 & 2 & 3 & 4 & 5 \\
\delta_{j} \approx & 0.5584 & 0.6937 & 0.4771 & 0.6058 & 0.7155
\end{array}
$$

whence we conclude that expert no. 3 had the highest concentration of evaluation effort.

\section{CONCLUSION}

We consider the approaches to making decisions for control problems in nonstandard situations with a lack of the previous experience and incomplete knowledge of the considered problem. In such cases we usually cannot do without expert evaluations which lead to the process of group decisionmaking, and it becomes necessary to solve a problem of alternatives aggregation. It has been proposed to solve such problems by means of fuzzy sets. The material of the paper is divided into two parts. In Part 1, an approach is proposed for the processing of quantitative expert evaluations which are used in the group decision-making. The approach is based on the coordination index and the similarity of finite collections of fuzzy sets and takes into account the specific character of the fuzzy aggregation operator. The approach is discussed in full detail and its algorithm is presented. An example of the application of the proposed method is given. 
The approach to making decisions for control problems is considered in nonstandard situations, in which there is a lack of the previous experience and the available information is uncertain and/or incomplete and/or weakly structured. In the framework of the proposed approach, quantitative expert evaluations are processed by using a special mathematical apparatus that employs the coordination index, the similarity of finite collections of fuzzy sets and takes into account the specific character of a fuzzy aggregation operator. The coordination index of a finite collection of fuzzy sets introduced in a new way forms the basis of the proposed approach. Setting aside the question of independence and completeness of the introduced postulates (Definition 2.3), by constructing the corresponding example [7] we prove that this axiomatics is consistent. The proposed approach is discussed in full detail and its algorithm is presented. An example of the application of the proposed method is given.

The prospects of the obtained results are briefly as follows. By virtue of (2.7) the proposed fuzzy aggregation method makes it possible to increase the coordination degree of experts' evaluations by $n S^{*} / \sum_{i=1}^{n} S_{i}$ times (here $X=\left\{x_{1}, x_{2}, \ldots\right.$ . , $\left.x_{n}\right\}, S_{i}$ are the coordination indices at each element of the universe, $S^{*}$ is a maximal coordination index among all oneelement fuzzy sets).

The method may also be helpful for a group of experts in the implementation of a project. Given a project with its vector of goals, the problem is to determine the coordination degree of evaluations made by contesters claiming to join the project group. The proposed approach enables one to calculate this degree and collect the most efficient project group.

\section{REFERENCES}

[1] A. Averkin, I. Batirshin, A. Blishun. V. Silov, V. Tarasov, Fuzzy sets in the Models of Control and Artificial Intelligence (Nauka Press, Moscow, 1986) (in Russian).

[2] D. Dubois, H. Prade, Possibility theory, Plenum, New York, 1988

[3] M.Meskon , M.Albert, F.Kheduri - Management $-3^{\text {rd }}$ Edition: translation from English- M.: LLC “I.D. Williams", 2007.-p.602.

[4] P.Schoderbek - Management Systems - 2nd ed - New York: Wiley, 1971, p.124.

[5] H.Simon - The Sciences of the Artificial. The MIT Press. ISBN:0-26269191-4; 978-0-262-6 9191-8; 1996.

[6] H.Simon, D.Smithburg,V.A. Thompson- Public Administration. Alfred A. Knopf:New York, 1961, p.493.

[7] T. Tsabadze, The coordination index of a finite collection of fuzzy sets, Fuzzy Sets and Systems 107 (1999) 177-185.

[8] T. Tsabadze, A method for fuzzy aggregation based on grouped expert evaluations, Fuzzy Sets and Systems 157 (2006) 1346-1361.

[9] J. Vaníček, I. Vrana and S. Aly, Fuzzy aggregation and averaging for group decision making: A generalization and survey. Knowledge-Based Systems 22 (2009) 79-84.

[10] L. A. Zadeh, Fuzzy sets, Inform. Control 8, No. 3 (1965), 338-353.

\section{Creative Commons Attribution License 4.0 (Attribution 4.0 International, CC BY 4.0)}

This article is published under the terms of the Creative Commons Attribution License 4.0 https://creativecommons.org/licenses/by/4.0/deed.en_US 